\title{
A Review on Shape based Descriptors for Image Retrieval
}

\author{
Pushpendra Singh \\ Research Scholar, \\ Dept. Of IT \\ IPEC, Ghaziabad, UP (INDIA)
}

\author{
V.K. Gupta \\ Professor, \\ Dept. of EC \\ IPEC,Ghaziabad,UP \\ (INDIA)
}

\author{
P.N. Hrisheekesha \\ Director \& Professor \\ Dr. K.N. Modi Institute of Engg. \\ \& Technology \\ Modi Nagar (UP) \\ (INDIA)
}

\begin{abstract}
In the age of information technology, a large number of images are generated at 24/7 which leads to a growing interest for searching out similar images from the large databases/ data warehouses. For searching an image from the database, images need to be described by certain features. The most important feature to describe an image is its shape. Now-adays, shape is used for image retrieval. Description of Shape is denoted by various techniques which are generally divided into two broad categories- Region based descriptor and Contour based descriptor. Contour based descriptor considers the whole area of the image while region based descriptor considers the boundary lines of the image. In this paper, shape based descriptors are reviewed. Some important shape descriptors have been identified for image retrieval according to the standard principles.
\end{abstract}

\section{General Terms}

Image Retrieval, Content Based Image Retrieval, Fourier Descriptor

\section{Keywords}

Content based image Retrieval (CBIR), Discrete Cosine Transform (DCT), Shape based Descriptor, Fourier Descriptor (FD), Wavelet Descriptor (WD).

\section{INTRODUCTION}

Due to rapid advancement of digital and information technologies, a huge amount of images are generated and digitally available around the globe. Locating the images becomes our primary goal to use these images for different applications. To accomplish this goal, content based image retrieval (CBIR)[16] has emerged as a multimedia application. To describe the images in CBIR their low-level features such as shape, color and texture or combination of them are used.

The most important low-level feature of an image is its shape. Any scene is considered as a collection of individual objects. The object of a scene can best be identified by its shape. A number of applications on shape retrieval can be found in literature in many areas like military, medical, law enforcement, object recognition etc. The techniques for shape based feature extraction can generally be classified into two broad categories-contour based method and region based methods. The contour based methods, such as Fourier Descriptors (FD)[21,20], Wavelet Descriptors(WFD), Curvature Scale Space Descriptor(CSSD) etc. uses the contour point only, while in region based methods such as Generic Fourier Descriptor(GFD), Geometric Moments Invariants and Algebraic Moment Invariants uses both contour points as well as interior points of a shape. In this paper, promising shape descriptors based on their retrieval performance are reviewed and identified. In section 2 , the review of literature on shape based descriptors is given.
Section 3 discusses some simple shape descriptors as well as some standalone shape based descriptors. This paper is concluded in section 4 .

\section{REVIEW ON SHAPE DESCRIPTOR}

Shape descriptors are classified into boundary-based and region-based methods. This classification takes into account whether shape features are extracted from the contour or from the whole shape region. Shape descriptors are further divided into structural (local) and global descriptors. If the shape is represented by segments or sections, it is structural and if it is from the whole shape region, it is global. Another classification categorizes the shape description into spatial and transform domain techniques, depending on whether direct measurements of the shape are used or a transformation is applied.

Rong-Xiang $\mathrm{Hu}$, et.al. [1] had proposed two novel shape descriptors, angular pattern (AP) and binary angular pattern (BAP), and a multi scale integration of them for shaperetrieval. Both AP and BAP are intrinsically invariant to scale and rotation. To integrate different scales of AP and BAP, the $\mathrm{z}$-score normalization was applied for distance matrices computed at each scale, and the normalized distance matrices were summed together to create a new distance matrix for shape retrieval. The Sequential Forward Selection (SFS) scheme was used to determine a set of scales that effectively capture discriminative information. More importantly, being global shape descriptors, the proposed shape descriptors were computationally very efficient, while possessing similar discriminability as state-of-the-art local descriptors. As a result, the proposed approach was attractive for real world shape retrieval applications. The experiments on the widely used MPEG-7 and TARI-1000 data sets demonstrated the effectiveness of the proposed method in comparison with existing methods. They had suggested three different ways to improve proposed approach, first to use different integration methods such as normalization procedure instead of z-score, second to use a more elaborate scale selection method and last to use a cross bin histogram distance similarity measure among different shapes.

R. Litman and A.M. Bronstein [2] had formulated a generic family of parametric spectral descriptors. They follow the spirit of Weiner Filter in signal processing and showed a learning scheme for the construction of optimized spectral descriptors and relate them to mahalanobis metric learning. The appropriateness of this descriptor was demonstrated on synthetic and scanned human images. They have performed their experiments on TOSCA and SCAPE datasets. The proposed descriptor was computed by applying a bank of filters to the shape's geometric features at the different frequencies and generalizes the heat and wave kernel signatures. Even mahalanobis metric leaning gave good 
results but they had suggested other metric learning approaches such as maximum-margin learning keeping in mind with a specific task such as ranking. They had suggested for real shape retrieval and matching task to use spectral feature descriptor to generate global shape descriptors by means of vector quantization or sparse coding for better retrieval performance.

Swati Agrawal, A.K. Verma and Preetvani Singh [3] proposed an algorithm based on discrete wavelet transform(DWT) and Edge Histogram Descriptor(EHD) feature of MPEG-7.The orientation of dominant edges was calculated with the help of wavelet coefficient's which will increase the performance of image retrieval [19,22,24,25] for shape and texture based search. The proposed algorithm was applied to Wang database and results were compared to various other techniques. The improvement in terms of precision and recall was found around $15 \%$ to $20 \%$. They had suggested to use multiple feature descriptors or advanced retrieving techniques to satisfy human perception in content based image retrieval process.

Subrahmanyam Murala, et al. [4], had proposed a new feature descriptor and implemented using local tetra patterns (LTrPs). The grey-level difference between referenced pixel and its surrounding pixels were computed using Local binary patterns (LBPs) and Local ternary patterns (LTPs).The Gabor transform was used to analyze the effectiveness of proposed descriptor. The descriptor's performance was compared with LBP, the local derivative patterns and the LTP using standard databases such as Corel 1000 database (DB1), Brodatz Texture database (DB2) and MIT Vistex database (DB3). The performance of new feature descriptor was improved by a reasonable amount in terms of average precision and average recall on the database. R.B. Yadav et al. [5] reported retrieval and classification of shape based objects employing three techniques-Fourier Descriptor (FD), Generic Fourier Descriptor (GFD) and Wavelet-Fourier Descriptor (WFD) and applied to a database of seven different types of shapes. The centroid distance based shape signature and the Euclidian distance for similarity measurement have been used by them. More than $95 \%$ performance was achieved for every class of objects by Wavelet-Fourier Descriptor which was much better than the performance of Fourier Descriptor and General Fourier Descriptor.

Deng Sheng Zhang, Guojun Lu, [6] had reviewed and evaluated different Fourier Descriptors(FDs) for image retrieval. They studied three methods- Conventional FDs, Affine FDs and Short time FDs. Their study was based on techniques of acquiring FD's retrieval effectiveness and compactness. Conventional FDs are based on applying Fourier Transform on a 1-D shape signature function. They have proposed two new shape signatures- Chord length signature and Area signature to improve existing signature methods. Various other signature methods are position function, centroid distance, cumulative angular function, curvature signature. By using any one of shape signature method FD is derived in which the set of magnitudes of the normalized Fourier co-efficient of the shape are used as Fourier Descriptors. They then studied the conventional, Affine FD and Short time FD. The convergence speed of each acquired FD was used to roughly estimate the number of FD features needed for shape description.

Retrieval experiments are conducted to test the retrieval effectiveness of different FDs using a standard shape database. Compactness study is followed to estimate the actual number of FD features needed for image description and retrieval. The study says that FD derived from centroid distance function and area functions are most suitable for image retrieval. FD derived from curvature function and cumulative angle function is not suitable for image retrieval due to very slow convergence speed. FDs derived from position function and affine function is not suitable for general shape retrieval due to sensitiveness to boundary changes. Although FD derived from chord length signature has satisfactory retrieval performance, but not desirable due to expensive computations and non robustness to noise.

Performance of FDs is higher than SFDs in terms of retrieval effectiveness and computation complexity but SFD is more accurate for local boundary features than FD. The study says that 10 FD features are sufficient for generic shape description.

\begin{tabular}{|c|c|c|c|c|}
\hline \multicolumn{5}{|c|}{ Table 1. Review On Shape Based Descriptors } \\
\hline S.No. & NAME OF DESCRIPTOR & $\begin{array}{ll}\text { NAME } & \text { OF } \\
\text { AUTHORS } & \\
\end{array}$ & $\begin{array}{l}\text { YEAR OF } \\
\text { PUBLICATION }\end{array}$ & $\begin{array}{l}\text { DESCRIPTION } \\
\text { TECHNIQUE/METHOD }\end{array}$ \\
\hline 1. & $\begin{array}{ll}\text { Discreminant } & \text { Face } \\
\text { Descripor(DFD) } & \end{array}$ & Zhen Lie et al.[2] & 2014 & Face Recognition \\
\hline 2. & $\begin{array}{lll}\begin{array}{l}\text { Binary } \\
\text { (BAP) }\end{array} & \text { Angular } & \text { Patterns } \\
\end{array}$ & $\begin{array}{l}\text { Rong-Xiang } \mathrm{Hu} e t \\
\text { al.[1] }\end{array}$ & 2014 & $\begin{array}{l}\begin{array}{l}\text { Region based global shape } \\
\text { descriptor }\end{array} \\
\end{array}$ \\
\hline 3. & $\begin{array}{l}\text { One Way Patch Descriptor } \\
\text { Based on DCT }\end{array}$ & Xiaobo Chen et al. & 2011 & Region Based Descriptor \\
\hline 4. & Local Binary Patterns(LBP) & Moore S. et al. & 2011 & Global Shape Descriptor \\
\hline 5. & $\begin{array}{ll}\text { Wave } & \text { Kernel } \\
\text { Signature(WKS) } & \\
\end{array}$ & M. Aubry et al. & 2011 & Laplacian Based \\
\hline 6. & $\begin{array}{ll}\text { Weber's } & \text { Local } \\
\text { Descriptor(WLD) } & \\
\end{array}$ & Chen J. et al. & 2010 & $\begin{array}{l}\text { Region Based Global Shape } \\
\text { Descriptor }\end{array}$ \\
\hline 7. & Local Ternary Patterns(LTP) & Tan X et al. & 2010 & $\begin{array}{lll}\text { Region Based Global Shape } \\
\text { Descriptor }\end{array}$ \\
\hline 8. & Heat Kernel Signature(HKS) & Sun et al. & 2009 & Laplacian based \\
\hline 9. & $\begin{array}{l}\text { Global point signature feature } \\
\text { descriptor }\end{array}$ & Rustamov & 2007 & Laplacian based \\
\hline 10. & $\begin{array}{ll}\text { Generic } & \text { Fourier } \\
\text { Descriptor(GFD) } & \\
\end{array}$ & Zhang and $\mathrm{Lu}$ & 2002 & Spectral Descriptor \\
\hline 11. & $\begin{array}{lcr}\text { Curvature } & \text { Scale } & \text { Space } \\
\text { Descriptor(CSSD) } & \\
\end{array}$ & $\begin{array}{l}\text { Abbasi et al. } \\
\text { Mokhtarian et al. }\end{array}$ & $\begin{array}{l}2000 \\
1996\end{array}$ & Spectral Descriptor \\
\hline 12. & Wavelet Descriptor & Tieang and Boles yang & 1997 & Spectral Descriptor \\
\hline
\end{tabular}




\begin{tabular}{|c|c|c|c|c|}
\hline & & et al. & & \\
\hline 13. & Fourier Descriptor & $\begin{array}{l}\text { Kauppinen et al. } \\
\text { Persoon and Fu }\end{array}$ & $\begin{array}{l}1995 \\
1977\end{array}$ & Spectral Descriptor \\
\hline 14. & $\begin{array}{l}\text { Shape Signature } \\
\text { (Chain Code, Centroid } \\
\text { distance, cumulative Angles, } \\
\text { Ecentricity and Circularity) }\end{array}$ & $\begin{array}{l}\text { Davies } \\
\text { Freeman and Saghri } \\
\text { Niblack et al. }\end{array}$ & $\begin{array}{l}1993 \\
1997 \\
1993 \\
\end{array}$ & Contour Based Methods \\
\hline 15. & $\begin{array}{l}\text { Moment Descriptors } \\
\text { (Geometric moments, } \\
\text { Legendre moments } \\
\text { Zernike moments } \\
\text { Pseudo-Zernike moments) }\end{array}$ & $\begin{array}{l}\text { Hu } \\
\text { Liao and Pawlak } \\
\text { Niblack et al. } \\
\text { Teague } \\
\text { The and Chin } \\
\text { Taubin and Cooper }\end{array}$ & $\begin{array}{l}1962 \\
1996 \\
1993 \\
1980 \\
1988 \\
1991\end{array}$ & Region Based methods \\
\hline
\end{tabular}

\section{IMPORTANT SHAPE DESCRIPTOR}

In this section we briefly discuss some of promising and standalone shape based descriptors which were used in literature and applied in many real applications.

\subsection{Simple shape descriptors:}

Shape based descriptors can be classified into two classes of methods: contour based methods and region based methods.
Both methods can be distinguished on the basis of whether shape features are extracted from the contour only or extracted from the whole shape region. Some of shape descriptors are area, circularity (perimeter ${ }^{2} /$ area), eccentricity (length of major axis /length of minor axis), major axis orientation and blending energy. These simple shape descriptors are only useful for those images which have large differences. Hence they are used as filters to eliminate false hits to differentiate these shapes.

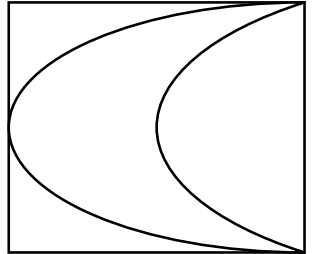

b

(a)

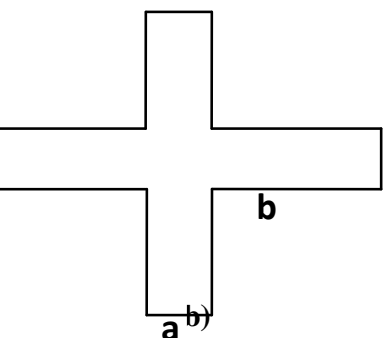

b

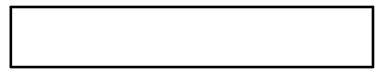

a

(c)

Figure 1. Shape Eccentricity and Circularity

In figure 1(a), since $a=b$, it does not correctly describe the shape. Hence for this type of shape circularity is a better descriptor. But in figure 1(b) and figure 1(c) $(a=2 b)$, since both figures have very different shapes so in this case, eccentricity is a better descriptor. Some of other simple descriptors are convexity, ratio of principle axis, circular variance and elliptic variance.

\subsection{Fourier Descriptors}

The French mathematician Jean Baptiste Joseph Fourier (born in 1768) stated that any periodic function can be expressed as the sum of sines and/or cosines of different frequencies, each multiplied by a different co-efficient. He also stated that aperiodic even functions ( But whose area under the curve should be finite) can be expressed as the integral of sines and/or cosines multiplied by the weighted function. This formulation was called Fourier transform. Both representations (Fourier series and Fourier transform) of a function have an important characteristic that a function can be reconstructed (recovered) completely via an inverse process, with no loss of information

A function $f(t)$ of a continuous variable $t$ that is periodic with period $\mathrm{T}$ can be represented as

$$
\begin{aligned}
f(t) & =\sum_{n=-\infty}^{\infty} c_{n} e^{j \frac{2 \pi n t}{T}} \ldots \ldots . \\
\text { Where } c_{n} & =\frac{1}{T} \int_{-T / 2}^{T / 2} f(t) e^{j \frac{2 \pi n t}{T} d t} .
\end{aligned}
$$

are the coefficients. This is called the Fourier series. The Fourier transform of $\mathrm{f}(\mathrm{t})$ mat be written as $f(\mu)=\int_{-\infty}^{\infty} f(t) e^{j 2 \pi \mu t} d t$

For any 1D function $\mathrm{f}(\mathrm{t})$, its Discrete Fourier transform is given by -

$$
a_{n}=\frac{1}{N} \sum_{t=0}^{N-1} f(t) e^{-j \frac{2 \pi n t}{N}}
$$

$$
\text { Where } \mathrm{n}=0,1,2, \ldots \ldots . \mathrm{N}-1 \text {. }
$$

This expression gives a set of Fourier co-efficients $\left\{a_{n}\right\}$ as a result, which can be a representation of a shape. According to D.Zhang and G. Lu [6], the set of magnitudes of the normalized Fourier co-efficient of the shape can be used as shape descriptors which are denoted as $\left\{F D_{n}, 0<\mathrm{n}<\mathrm{N}\right\}$. The city block distance $d$ between these FDs gives the similarity measure between a query shape $\mathrm{Q}$ and a target shape T. Arbter et al. proposed the Affine Fourier Descriptor (AFD) which was invariant under affine transformation. Since FD extracts both global as well as local features on shape boundary, Eichman et al. proposed Short-Time Fourier transform to locate local boundary features. It can be represented as -

$a_{n m}=\frac{1}{T} \sum_{t=0}^{N-1} f(t) g\left(t-n t_{0}\right) e^{-j \frac{2 \pi m t}{T}} d t$

Where $t_{0}$ is the filter step size. The window function (called Analysis Filter) is non-zero in the interested (local) region. 
Generally it is a Gaussian function or rectangular function. These above Fourier descriptors are contour based descriptors. The region based Fourier descriptors are called Generic Fourier Descriptors (GFD) and can be used for reconstruction and classification of object shapes. The GFD can be derived by applying a discrete 2D Fourier transform on a polar raster sampled shape image.

$$
D(\rho, \theta)=\sum_{r} \sum_{r} f\left(r, \theta_{i}\right) e^{\left[-j 2 \pi\left(\frac{r}{R} \rho+2 \pi \frac{i}{T} \emptyset\right)\right]}
$$

Where $0 \leq \mathrm{r} \leq \mathrm{R}$ and $\theta_{i}=i\left(\frac{2 \pi}{T}\right)(0 \leq \mathrm{I} \leq \mathrm{T}) ; 0 \leq \rho \leq \mathrm{R}, 0 \leq \emptyset$ $\leq \mathrm{T}$.

$\mathrm{R}$ and $\mathrm{T}$ are radial and angular resolutions and $\mathrm{f}(\mathrm{x}, \mathrm{y})$ is a binary function in shape application.

\subsection{Wavelet Fourier Descriptor(WFD):-}

The Wavelet transform's co-efficient of shape boundary are called Wavelet descriptors, which is a multi-resolution transformation for decomposing the signal object into different levels. WDs are used for extraction of local features of the decomposed sub-images. Hence, Wavelet Transform (WT) of an image boundary $z(t)$ can be represented as -

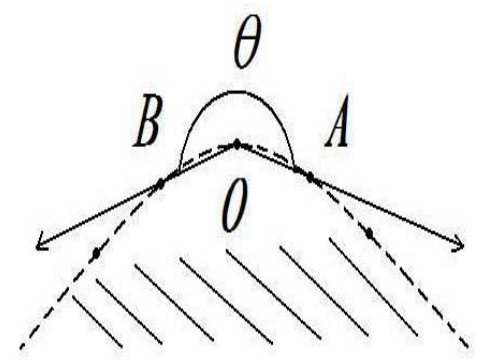

\section{Figure 2. Illustration of Angular Pattern (AP)}

Let there are $\mathrm{n}$ uniformly sampled contours, geometric features (e.g. curvature and distance to the centroid) used as a shape descriptors as shown in Figure 2. Since curvature and distance both are not invariant to scale and rotation transformation. Hence let a point $\mathrm{O}$ on the contour and $\mathrm{A}, \mathrm{B}$ is the two equidistance points from $\mathrm{O}$ before and after $\mathrm{O}$ respectively on the contour. The angle $\theta$ (anticlockwise) between $\mathrm{OA}$ and $\mathrm{OB}$ is used to describe the geometric properties of $\mathrm{O}$. This is called the angular feature which was extended to multi-scale. Suppose there are $\mathrm{S}$ number of points (As and Bs) away from $\mathrm{O}$ in each side where $1 \leq s \leq$ floor $\left[n-\frac{1}{2}\right]$. Then define angle $\theta$ s between OAs and OBs. For a given shape of $n$ contour points and a specific $s$, total $n$ angles can be drawn, which captures the geometric property of a shape at scale s. this representation is called the Angular Pattern (AP) of a shape.

These angular features are used for efficient shape retrieval using the histogram of APs. The histogram of APs can be generated by the following steps:

Step I. Given a contour of $\mathrm{n}$ points and a specific s, we extract $\mathrm{n}$ angular features, i.e.

$$
\text { APs }=\{\operatorname{APs}(i), i=1,2, \ldots \ldots \ldots n\}
$$

$$
c_{a}(b)=\frac{1}{\sqrt{|a|}} \int_{R} z(t) \emptyset\left(\frac{t-b}{a}\right) \mathrm{dt} .
$$

The equation gives the wavelet coefficients at the boundary of $\mathrm{z}(\mathrm{t})$ at a scale $\mathrm{a}$ and position $\mathrm{b}$. The selected coefficients of the Fourier transform of the wavelet coefficients $c_{a}(b)$ are called Wavelet Fourier Descriptor (WFD). They are defined as -

$F^{a}(k)=\frac{1}{N} \sum_{b=0}^{N-1} c_{a}(b) e^{\left(-j \frac{2 \pi b}{N}\right)}$

The Wavelet Fourier Descriptors are frequency domain descriptors classified as Low and High frequency descriptors. The details of general features can be represented by Low frequency descriptors while the finer features of an object can be represented by High frequency descriptors.

\subsection{Angular Pattern and Binary Angular Pattern}

Rong-Xiang, et al. [1] has proposed two novel shape descriptors as global descriptors. Both the descriptors utilize the angular information among contour points as patterns. Both the descriptors are invariant to scaling and rotation transformation.

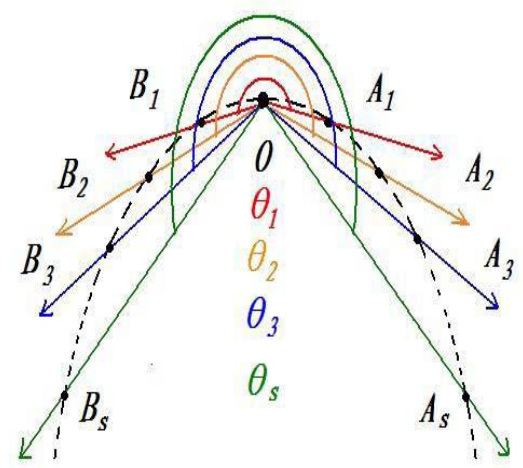

Figure 3. Illustration of multi-scale AP

Step II. Uniformly divide $[0,2 \pi]$ into K bins.

Step III. Construct the Histogram-

$$
\mathrm{H}=\{\mathrm{h}(\mathrm{k}), \mathrm{k}=1,2,3 \ldots \ldots, \mathrm{K}\}, \text { with each }
$$

bin as

$\mathrm{h}(\mathrm{k})=\operatorname{card}\left\{\mathrm{a} \mid \mathrm{a} \in A P s, \frac{k-1}{K} .2 \pi<a \leq \frac{k}{K} .2 \pi\right\}$,

$\mathrm{k}=1,2,3 \ldots ., \mathrm{K}$.

To compute two AP histograms-

$\mathrm{D}(\mathrm{Hs} 1, \mathrm{Hs} 2) \equiv \frac{1}{2} \sum_{i \leq k \leq K} \frac{\left[[h s 1(k)-h s 2(k)]^{2}\right]}{h s 1(k)+h s 2(k)}$,

where $\mathrm{hs} 1(\mathrm{k})$ and $\mathrm{hs} 2(\mathrm{k})$ are the kth bin of corresponding histogram and $\mathrm{K}$ is the number of histogram bins. Since $1 \leq$ $s \leq$ floor $\left[n-\frac{1}{2}\right]$, we can construct floor $\left[n-\frac{1}{2}\right] \quad$ AP histograms and each of them can be used to retrieve shape independently.

The simplest BAP is illustrated in Figure 3. Given a point $O$ on the contor and its equal distance point pairs $(A, B)$ and $(C$, $D$ ), a segment containing five points can be constructed. Obviously, three angular features, i.e., $\theta_{O}, \theta_{A}$ and $\theta_{B}$ can be calculated. Then, $\theta_{A}$ and $\theta_{B}$ are compared with $\theta_{O}$. If the angle $\theta_{A}$ or $\theta_{B}$ is larger than $\theta_{O}$, the corresponding point is coded with 1 , otherwise, it will be coded with 0 . Consequently, for this segment with five points, a two-bit binary pattern can be 
generated, which is robust to a small degree of non-rigid distortion. As shown in Figure 4 and Figure 5, for $\theta_{O}=\pi$, there are two cases. First, $\theta_{A}$ and $\theta_{B}$ are greater than $\theta_{O}$, and in this situation the BAP of point $O$ is always coded as [1 1] for all three deformed contours depicted in Figure 4. Similarly, the BAP of point $O$ in Figure 5 is [0 0 ] for all three contours for the second case in which both $\theta_{A}$ and $\theta_{B}$ are smaller than $\theta_{O}$

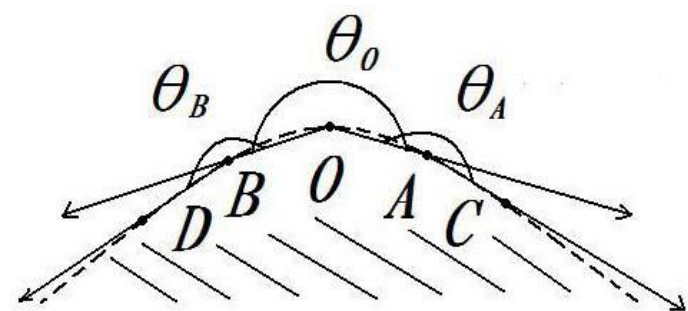

Figure 4. Illustration of the simplest BAP

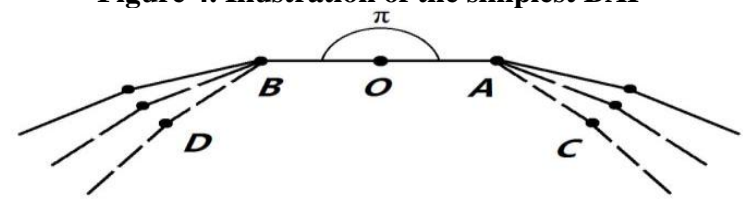

(a)

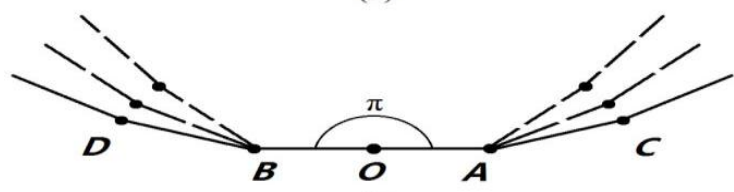

(b)

Figure 5. Illustration of the BAP feature to small degrees of non-rigid distortion which does not affect the binary angular relations: (a) all patterns are [1 1]; (b) all patterns are $\left[\begin{array}{ll}0 & 0\end{array}\right]$

Since AP is multi-scale, the proposed simplest BAP can also be extended to multi-scale. If the pattern is constructed with $O, A s, B s, C s$ and $D s$ as defined before, then the computed two-bit binary pattern will capture another scale of angular relations. Finally, floor $((n-1) / 2)$ scales of BAP with two-bit pattern can be extracted for a contour containing $n$ points.

\section{OTHER SHAPE DESCRIPTORS}

In the literature, a number of other shapes based descriptors was proposed and implemented on several applications. One of them is one way patch descriptor which was proposed by Xiaobo Chen et al. and based on discrete cosine transform (DCT) for real time patch matching. This descriptor improves speed of patch matching without losing accuracy and robustness between the patches. The authors suggested that this descriptor can also be useful for object detection and pose estimation applications. David Edmundson and Gerald Schaefer proposed a very fast method for JPEG compressed image retrieval. Their method was based on Huffman tables contained in JPEG header. This header can be optimized on per image basis which improves compression rate and gives a very useful descriptor. Since JPEG compression is based on DCT so for image retrieval, Huffman tables uses DCT coefficients for feature extraction. (Mokhtarian et al., 1996[]) proposed curvature scale space (CSS) descriptors which were based on key local shape features. This descriptor was proposed in MPEG-7 as a contour based descriptor. D. Zhang and Guojun Lu, 2003 [8] compared the two descriptorsFourier descriptor and CSS descriptor and found that FD performs better than CSSD in terms of robustness, low computation, hierarchical representation, retrieval performance and suitable for efficient indexing.

\section{CONCLUSION}

In this paper, existing shape descriptors have been reviewed. Generally these are of two types-contours based and region based. Both the types can further be divided into global shape descriptors versus local shape descriptors. Both the methods can work in space domain as well as transform domain.

Contour based methods have an edge over region based methods in popularity due to the following reasons-

i. Humans can easily discriminate shapes by their contours.

ii. In many applications, the contour of a shape has importance not its interior content.

These methods also have some limitations-

i. $\quad$ Contour based shape descriptors are sensitive to noise and variations due to use of small parts of shapes.

ii. In many applications, contours are not available.

iii. Some of the applications have interior contents more importance.

Region based methods can overcome these limitations. They are more robust and can be applied in general applications. They can cope with shape defection. In the review we have found that spatial domain methods have two drawbacks: noise sensitivity and high dimension. The remedy of these can be histograms, moments, scale space and spectral transform. Among all of them spectral transform are most promising. Shape representation using Fourier or Wavelet Fourier Descriptor is simple to compute, robust and compact. Generic Fourier Descriptors (GFD) performs very well when some significant part of the shape is missing or occluded.

To summarize, local methods are useful where partial matching is required. For general applications, spectral transform methods are the best choices

\section{REFERENCES}

[1] Rong-Xiang Hu, et.al. , "Angular Pattern and Binary Angular Pattern for Shape Retrieval", IEEE Transactions on Image Processing, Vol. 23, No. 3, pp. 1118-1127, March 2014.

[2] Zhen Lei,et.al. , "Learning Discriminant Face Descriptor" IEEE Transactions on Pattern Analysis and machine Intelligence, Vol. 36, No.2, pp. 289-302, FEBRUARY 2014

[3] R. Litman and A.M. Bronstein, "Learning Spectral Descriptors for Deformable Shape correspondence", IEEE Transactions On Pattern Analysis And Machine Intelligence, Vol. 36, No. 1, pp. 171-180, January 2014.

[4] Swati Agrawal, A.K. Verma and Preetvani Singh, "Content based image retrieval using Discrete Wavelet transform and Edge Histogram Descriptor", International Conference on Information Systems and Computer Networks, pp. 19-23, 2013.

[5] Subrahmanyam Murala, R.P. Maheshwari and R. Balasubramanian, "Local Tetra Patterns: A New Feature Descriptor for Content based image retrieval", IEEE Transactions on Image Processing, Vol. 21, No. 5, pp.2874-2886, May 2012. 
[6] R.B. Yadav, et.al. , "Retrieval and Classification of shape based objects using Fourier, Generic Fourier and Wavelet-Fourier descriptors technique: A Comparative Study", Elsevier's journal of optics and lasers in engineering, Vol.45, pp.695-708, 2007.

[7] Deng Sheng Zhang, Guojun Lu, "Study and Evaluation of different Fourier Methods for image retrieval", Elsevier journal on image and vision computing, Vol. 23, pp.33-49, 2005.

[8] Deng Sheng Zhang, Guojun Lu, "Review of shape representation and description techniques", Elsevier Journal of the pattern recognition society, Pattern Recognition, Vol.37, pp.1-19, 2004.

[9] Deng Sheng Zhang, Guojun Lu, "A Comparative Study of curvature scale space and Fourier Descriptors for shape based image retrieval", Elsevier's journal of Visual Communication and image representation, Vol. 14, pp.41-60, 2003.

[10] Lele Zhao, Bing Wang, Huazhong Shu, "Multi-Scale Fourier Descriptor with phase information for image retrieval", Elsevier's Journal of Energy Procedia, Vol. 13, pp.5068-5075, 2011

[11] Chien-Cheng Tseng and Shyi-Chyi Cheng, "Digital color image sharpening using fractional differentiation and discrete cosine transform", International symposium on Communication and information technologies, pp.181186, 2012.

[12] B.H. Shekar, et.al. , "Face Recognition based on Fractional Discrete Cosine Transform", International Conference on Recent Trends in Information Technology, pp.987-991, June 3-5, 2011.

[13] P.S Hiremath and Jagadeesh Pujari, "Content based image retrieval based on color, Texture and Shape features using image and its complement", International Journal of Computer Science and Security, Vol. 1, Issue 4, pp.25-35, 2009

[14] Xiaobo Chen, Feng Ye, Fuguo Zhu, Aidong Men, "Real -Time Affine invariant patch matching using DCT and Affine space quantization", $18^{\text {th }}$ IEEE International Conference on Image Processing, pp.2993-2996,2011.

[15] Soo-Chang Pei and Min-Hung Yeh, "The Discrete Fractional Cosine and Sine Transforms", IEEE
Transactions on signal processing, Vol. 49, No. 6, June 2001.

[16] G. Rafiee, S.S. Dlay and W.L. Wro, "A review of content based image retrieval", $7^{\text {th }}$ International Symposium on Communication system networks and digital signal processing, pp.775-779, 21-23 July, 2010.

[17] B.H. Shekar, et.al. , "Face Recognition based on Fractional Discrete Cosine Transform", International Conference on Recent Trends in Information Technology, pp.987-991, June 3-5. 2011.

[18] Mussarat Yasmin, et.al., "Content Based Image Retrieval by Shape, Color and Relevance Feedback", Life Science Journal, 10(4s), pp. 593-598, 2013;

[19] Ot'avio A. B. Penatti, et.al. "Comparative Study of Global Color and Texture Descriptors for Web Image Retrieval”, Journal of Visual Communication and Image Representation, pp.1-53, September 16, 2011.

[20] Maruti Agrawal, et.al. , "A mirror reflection and aspect ratio invariant approach to object recognition using Fourier descriptor", Elsevier's Journal of Applied Soft Computing, Vol. 11, pp.3910-3915, 2011.

[21] Lele Zhao, Bing Wang, Huazhong Shu, "Multi-Scale Fourier Descriptor with phase information for image retrieval", Elsevier's Journal of Energy Procedia, Vol. 13, pp.5068-5075, 2011.

[22] Jian Feng Wang and Xiaorong Zhao, "A New approach for image retrieval with integrated Euclidean Distance and rotational correlation, IEEE Explore, 2011.

[23] Bin Zhang, Kuizhi Mei and Nannin Zheng, "Reconfigurable processor for binary image processing", IEEE Transactions on Circuits and Systems for Video Technology, Vol. 23, No. 5, pp. 823-831, May, 2013.

[24] B. Syam, Sharon Rose Victor J, Y. Srinivasa Rao, "Efficient similarity measure via Genetic Algorithm for content based medical image retrieval with extensive features", IEEE Transactions, pp.704-711, 2013.

[25] B. Verma Jyothi, C.Uma Shanker and S. Madhusudhan Verma , "Reseach study of neural networks for image categorization and retrieval", IEEE Transactions Vol. 2, pp.685-690, 2012. 